\title{
The Perils of Haemorrhoids Treatment
}

\author{
Abdel Latif K Elnaim ${ }^{a}$, Wong M.P.K. ${ }^{a}$, Sagap $I^{a}$ \\ ${ }^{a}$ Colorectal Unit, Department of Surgery, Universiti Kebangsaan Malaysia Medical Centre, Kuala Lumpur, \\ Malaysia
}

\section{ABSTRACT}

Treating problematic haemorrhoids has taken a long turmoil route. Its peak incidence is among 45 to 65 years of age group. Typically, problematic haemorrhoids present in multi-symptoms forms like a prolapsed lump, painless bleeding, discomfort, soiling, or itchiness. Many theories were postulated in the pathophysiology of symptomatic haemorrhoids. The sliding and engorged of anal cushion with hypervascularity is the most popular. This is an updated review of published English-language literature regarding the treatment of haemorrhoids. The treatment includes medical therapy, office procedures, and surgical operations. Merits and demerits of the different modalities of treatment of haemorrhoids are presented. The best treatment options are difficult to ascertain. It should be tailored to individualize treatment according to their presentation and severity. Up till recently, the excisional haemorrhoidectomies are considered the standard procedure for haemorrhoid treatment. These techniques produce significant post-operative pain to the patient, which hinders them from normal daily activity. Recent advancement in surgical intervention has focused on minimising severity of pain and enhances recovery.

KEYWORDS: haemorrhoids, haemorrhoidectomy, haemorrhoidopexy, haemorrhoidoplasty

\section{INTRODUCTION}

\section{The Evolution of Haemorrhoids Surgery}

Haemorrhoids treatment has been described since 1700 BC by the Egyptian papyrus scriptures and 460 BC by Hippocrates. Fredrick Salmon was thought to be the first to be the leader of modern haemorrhoids treatment. In 1888, Salmon proposed a combination of ligation and excision for haemorrhoids treatment. The techniques described by Milligan-Morgan and Ferguson are considered a modification of his technique. In 1937, Milligan and Morgan introduced Excisional haemorrhoidectomy in the United Kingdom which includes excision of haemorrhoidal tissue and vessels with the placement of a suture at the haemorrhoid pedicle, and the wound left opened to heal by secondary intention. In 1952, Ferguson further modified this technique by completely

Corresponding Author:

Assoc. Prof. Datuk Dr. Ismail Sagap

Colorectal Unit,

Department of Surgery,

Universiti Kebangsaan Malaysia Medial Centre,

Kuala Lumpur, Malaysia.

Tel No: +603-9145 6201/6202

Email: drisagap@yahoo.com close the wound by a continuous suture in United States. ${ }^{1-3}$

As time progresses, several newer techniques have developed. In the 1960s, Senagore and associates utilised laser therapy in haemorrhoid treatment. It involved the application of laser probe within the vascular pedicle. ${ }^{4}$ Doppler-guided localization and trans-fixation of haemorrhoidal arteries with or without haemorrhoidopexy was introduced by Morinaga in 1995.,6 Later, the use of a circular stapling device was proposed by Longo in 1998 aiming to replace the haemorrhoidal cushion to its original position hence restoring its physiological function. ${ }^{7}$ In the meantime, radiofrequency ablation is an innovative procedure designed by Gupta in 1998 for treatment of grade III and IV haemorrhoids. The procedure uses an Ellman dual-frequency, 4-MHz radiofrequency generator used to ablate the haemorrhoidal tissues. ${ }^{2,8}$ Following this principle, Doppler guided-haemorrhoidal arterial ligation is further modified by Vincent et al. with the concept of haemorrhoidal artery embolisation, which then termed as the emborrhoid technique. ${ }^{9}$ 
Definition, clinical presentation, classification, and pathophysiology

Haemorrhoids are vascular cushions composed of fibro-elastic tissue, muscle fibers, and vascular plexuses with arteriovenous anastomoses. ${ }^{2,6}$ Symptomatic haemorrhoids are mainly presented as prolapsed lump, which may require manual reduction. Classification is based on their site and degree of prolapse. Internal haemorrhoids are localised above the dentate line and covered by columnar epithelium. These had been conveniently classified by Goligher et al. as grade I haemorrhoids which is anal cushions that bleed without prolapse, grade II haemorrhoids with anal cushions prolapse on straining but reduce spontaneously, grade III haemorrhoids with anal cushions prolapse on straining or exertion and require manual reduction and the Grade IV haemorrhoids with irreducible prolapse. ${ }^{2,10,11}$ On the other hand, external haemorrhoids starts below the dentate line and covered with squamous epithelium. ${ }^{10}$ Other presentations include painless bleeding, discomfort, discharge, hygiene problems, soiling, and pruritus. ${ }^{6,10,11}$

The pathophysiology of haemorrhoids remains poorly understood. The universal theory is the pathological loosening and disintegration of supporting connective tissues in anal cushions, which causes an abnormal downward displacement and venous dilatation. This is commonly known as the sliding anal canal theory. ${ }^{12}$ This occurred when the supporting structure around the anal cushion was disrupted. The avascular supporting structure of the anal cushion is also known as the muscle of Treitz, which consists of the anal submucosal muscle that interlinked between the sinusoids that attach the anal cushion to the haemorrhoids floor, and together with the Park's mucosal suspensory ligament that penetrates and adhered it to the internal anal sphincter. ${ }^{13}$ It recoils the anal cushion back to its original position after propulsion of faeces during defaecation.

Another theory is hyperperfusion of the haemorrhoidal arteriovenous plexus which form varicosity. ${ }^{10,14}$ This theory has ceased its popularity as there is no increased prevalence of haemorrhoids among those with portal hypertension. ${ }^{12}$ Possible contributing factors include lack of dietary fibre, prolonged straining, excessive commode time, constipation, diarrhoea, pregnancy, sedentary lifestyle, and strong family history. None of these aetiologies is supported with good evidence apart from pregnancy. ${ }^{2,10}$

\section{Treatment options}

\section{Conservative (Medical) Treatment}

\section{Lifestyle modification}

Dietary and behavioural modifications are among the first-line recommendations for patients with haemorrhoids. Standard recommendations include increasing dietary fibre intake, the avoidance of straining and minimising time on the toilet during defaecation, and usage of soothing sitz baths several times per day. ${ }^{8,15,16}$

\section{Drug therapy}

Many topical agents were recommended for symptomatic control in haemorrhoids. A combination of lignocaine with steroids and keratolytic agents are commonly used without strong evidence of permanent relief. ${ }^{8,15}$ Micronized purified flavonoid has been recommended extensively in symptomatic haemorrhoids. ${ }^{17}$ This is thought to improve the venous tone and the lymphatic flow with reduction of inflammation. Recent study on flavonoid shows that it is highly effective in the treatment of acute haemorrhoids; to reduce the duration of bleeding and the resolution of anal pain resulting from prolapsed haemorrhoids. ${ }^{18}$ Unfortunately, flavonoids are to be used in high doses and in a long duration of time to produce effective treatment outcome for prolapsed haemorrhoids..$^{8,15,19,20}$ In addition, a topical anti-inflammatory preparation of Pileseptine-e with strong osmotic properties has been introduced with an acceptable outcome. It is applied as a film layer that reduced the size by fluid exudation to control symptoms. $^{21}$

\section{Rubber Band Ligation}

Rubber band ligation is the most accessible treatment for bleeding internal haemorrhoids. Single or multiple ligations can be performed. Bleeding, perineal pain, vaso-vagal symptoms, slippage of bands, priapism, obstructive urination, anal fissure, and chronic longitudinal ulcers are among 
the minor complications frequently encountered. However, massive bleeding, thrombosed haemorrhoids, severe pain, urinary retention needing catheterisation, and pelvic sepsis are among the uncommon major complications. In addition, several infectious complications have been reported, including pelvic sepsis, Fournier's gangrene, liver abscesses, tetanus, and bacterial endocarditis. One of the major concern for RBL is post-procedure bleeding, especially among those who are on antiplatelet and anticoagulant..$^{22}$ In a retrospective study by Nelson et al., the bleeding after RBL usually occurs after 5-10 days during which the mucosal sloughed off. ${ }^{23}$ In their study, they found out that those who had antithrombotic agent withheld after seven days post-procedure has the same bleeding risk as those who are not on antiplatelet therapy. In their cohort, they noticed those who are on clopidogrel tends to bleed later which is after 9.5 days after the procedure. ${ }^{23}$ Therefore, they recommended after RBL the anticoagulant should be withheld for seven days and antiplatelet for ten days to reduce risk of postprocedure bleed. ${ }^{23} \mathrm{RBL}$ offers 1 -year results with an estimated $50 \%$ to $70 \%$ success rate. ${ }^{22,24}$

\section{Injection Sclerotherapy}

Sclerosant injection using almond oil, arachis oil, sodium morrhuate or quinine urea, polidocanol and pheno-alcohol is thought to be simple, safe and produces rapid results. It can also be applied via the endoscopic procedure. However, it is inferior to rubber band ligation in terms of rapid resolution of bleeding and complications such as massive bleeding, intractable pain, and prostatic symptoms are among the many encounters due to technical inaccuracy. In a large prospective study by Fernandes et al., an observation of 2000 participants with $10.5 \%$ participants on antithrombotic therapy, reported that the injection sclerosant therapy is safe with no significant increased bleeding risk when compared to those without antithrombotic therapy. ${ }^{25}$ The recurrence rate with this procedure has been reported as 5 $10 \%{ }^{26,27}$

\section{Infrared photocoagulation}

Infrared photocoagulation is an effective office procedure suitable for first and second-degree haemorrhoids. The infrared probe is applied to the pedicle of the haemorrhoids through a proctoscope to produce a circular burn with a depth of $2 \mathrm{~mm}$. The recommended exposure for each site is around 1 second. The procedure produced similar operative outcome when compared to banding and sclerotherapy with the advantage of being less painful. $^{2,8,19}$ When compared to excisional haemorrhoidectomy the prevalence of bleeding was $5.0 \%$ in the IRC group and $30.0 \%$ in the haemorrhoidectomy group, while recurrence was higher $20 \%$ versus $5 \%{ }^{28}$ Two randomized control trials was found the reported success rates of $67 \%$ and $96 \%$ of this procedure. ${ }^{10}$

\section{Radiofrequency ablation}

Radiofrequency ablation is an innovative procedure designed in 1998 by Gupta for grades III and IV haemorrhoids. The procedure uses the Ellman dualfrequency, $4-\mathrm{MHz}$ radiofrequency generator for the ablation of haemorrhoids. The alternating currents generate differences in the direction of the ions within tissue fluid that creates ionic instability and frictional heating, leading to coagulative necrosis of tissue. It presents better results in terms of postoperative pain and bleeding than stapled haemorrhoidopexy and Doppler-guided haemorrhoidal artery ligation. ${ }^{2,8,29}$

\section{Cryosurgery}

The cryosurgery uses a very low temperature creating water crystals within the cells resulting in the destruction of the cell membrane and tissues. The expected less pain effect from cryosurgery by freezing the sensory nerve endings and causing an immediate anaesthetic effect yields a completely opposite the desired clinical outcome. In addition to the lengthy procedure; other disadvantages include profuse discharge, prolonged recovery, and late return to work. Thus, cryosurgery does not seem to offer further advantages over other office procedures. ${ }^{10,30}$ No recent encouraging results were found, as its use regressed with time.

\section{Laser therapy}

The laser ablative treatment for haemorrhoids seems to have minimal complications. The beam causes tissue shrinkage and degeneration at different depths depending on the irradiance and the duration 
of laser light application. Recent evidence has reported that the application of laser technology in the treatment of haemorrhoids was safe, effective, and painless, and resulted in partial to complete resolution within a short time. Nd-YAG laser (neodymium-Yttrium-aluminium-garnet laser) is the most frequently used type for surgical intervention. ${ }^{4,28,31}$ Success rate in laser treatment can reach more than $95 \%$ in some studies with a similar number to patient satisfaction. ${ }^{2}$ With regards to pain score; it is very low compared to excisional procedures. $^{32}$ The recurrence rate after laser haemorrhoidoplasty was reported as $7.8-34 \%{ }^{33,34}$ In most of the laser haemorrhoidoplasty study, they recognised that this procedure is costlier than the rest of the haemorrhoid treatment procedures. Nevertheless, this procedure is easy to learn, quick to perform and produces rapid, effective short-term resolution of symptoms. ${ }^{4,32,34,35}$

\section{Conventional Haemorrhoidectomy}

Parks introduced submucosal haemorrhoidectomy procedure in 1956. It was introduced to reduce postoperative pain and avoid anal and rectal stenosis. Parks technique includes performing haemorrhoidectomy with preservation of the anal canal mucosa and reducing the surgical wound dimensions, hence, leading to shorter healing time, as well as lower stenosis index than the conventional techniques. As the mucosa is not included in the ligation, it reduces postoperative pain. However, the operative time is longer, the recurrence rate is higher and greater risk of bleeding during intraoperative and postoperatively. ${ }^{2,8,10,36}$

Excisional haemorrhoidectomy involves excision of the haemorrhoidal cushions and has generally been advocated for larger symptomatic haemorrhoids (grades III and IV). The technique involves the excision of haemorrhoid tissue. This was initially done with sharp dissection using scissors, which was then modified to the use of energy device to control bleeding $^{16,37}$.

In diathermy haemorrhoidectomy, coagulation occurs at temperatures higher than 150 Celsius. The formation of an eschar seals the bleeding area. Compared with conventional scissors haemorrhoidectomy, diathermy haemorrhoidectomy is associated with less bleeding, shorter operative time and lower postoperative analgesic requirement, but similar postoperative pain. ${ }^{10,38}$

The Ligasure haemorrhoidectomy offers promising haemostatic control and negates the need for pedicles ligation. Improved hemostasis also offer better visibility and therefore, accurate dissection. It is superior to conventional scissors and diathermy haemorrhoidectomy in terms of operative time, postoperative pain, urinary retention, and the time return to work. Although early functional and symptomatic outcomes have been satisfactory, longterm follow-up of patients following is necessary. ${ }^{3,37}$ There are several randomised trials to date comparing harmonic scalpel haemorrhoidectomy (HSH) with other various open and closed techniques. However, the results were inconsistent. Some studies showed an obvious superiority of $\mathrm{HSH}$ with respect to operative time, postoperative pain, blood loss, length of hospital stay, and time of returning to regular activity. However, its costeffective is still debatable. . $^{2,8,10}$

In summary, excisional haemorrhoidectomy is regarded as the ultimate standard in the treatment of symptomatic haemorrhoids with the least recurrence. Unfortunately, while being effective, it is nonetheless associated with severe immediate postoperative pain. ${ }^{24,39}$ The pain could last for weeks and may be associated with delayed return to work and normal daily activities. Furthermore, excisional haemorrhoidectomy may cause troublesome anal stricture complication and incontinence. $^{40}$

\section{Haemorrhoidal pedicle treatment}

Farag in 1978 (Farag procedure) introduced this procedure in which three interrupted sutures are used to interrupt the blood flow to the prolapsed haemorrhoids. However, this technique and its modifications were not favourable because of the immediate postoperative painful congestion resulting from interruption of the blood flow to the haemorrhoidal cushions. However, the prolapsed piles after this surgery will have a gradual shrinkage. ${ }^{10}$

Anopexy is a more straightforward technique for the treatment of advanced haemorrhoidal disease. It targets to control of bleeding, reduction of haemorrhoidal prolapse, and fixation of the 
haemorrhoid cushions to the underlying tissues. A stitch placed at the pedicle of the haemorrhoidal cushion significantly reduces the blood flow to the haemorrhoidal plexus. This is followed by fixation of the haemorrhoidal cushions to the underlying internal sphincter to oppose the prolapse. ${ }^{10}$

In Doppler-guided haemorrhoidal artery ligation (DG -HAL), the aim is to interrupt the blood supply to the haemorrhoids through multiple ligations of the branches of the inferior haemorrhoidal arteries identified by a Doppler device mounted on an operating proctoscope. The procedure is simple and has a short learning curve. As there is no wound, and the sutures were applied above the dentate line, pain is theoretically less and recovery enhanced. Despite the theorem, the pain tends to be moderate and resolved first few days after surgery such that there is minimal to no pain by 1-3 weeks. Minor complications include bleeding, urinary retention, and thrombosis and fissure formation. . $^{2,84}$

Following the same principle as DG-HAL, Vincent et al. proposed haemorrhoidal artery embolisation, termed the emborrhoid technique. This technique involves occlusion of haemorrhoidal arteries using an endo-vascular coil. This technique has several advantages: the haemorrhoidal tissue or cushion are left in place, preserves anal continence by reducing the risk of anal sphincter injury, and minimal bleeding as it does not involve the creation of rectal wounds. In a large randomised study found that DG-HAL has a clinical success rate of $82 \%$ with $30 \%$ recurrence at one year. ${ }^{9}$

Longo introduced stapled haemorrhoidopexy in 1998 using Procedure for the Prolapsed Haemorrhoid (PPH) device. It involves removal of a ring of the redundant rectal mucosa or expanded internal haemorrhoids. The principle is to pull and fix the prolapsed haemorrhoid tissue back up to its normal position within the anal canal and to disrupt the arterial inflow that traverses the excised segment. The advantages of PPH were shorter operating time, less postoperative pain, less postoperative urinary retention, and an earlier return to normal activity. The long-term recurrence is not as good as the excisional haemorrhoidectomy. It was reported that the recurrence rate for stapled haemorrhoidopexy was about 5.7-20.7\% compared to $0-3.9 \%$ in excisional haemorrhoidectomy. ${ }^{40,42,43}$ In addition, stapled haemorrhoidopexy has set-back of residual skin tags, which may have negative satisfaction to patients. ${ }^{2,3,7}$ A systematic review shows the stapler haemorrhoidopexy is comparatively cheaper than the conventional haemorrhoidectomy when calculating the disposable items, operation cost and hospital stay. However, the differences were not statistically significant. ${ }^{43}$

\section{Summary of the review}

\section{Are we adding misery to the problem? Are we helping the patients?}

Treatment of the haemorrhoidal disease is primarily aimed at addressing symptoms. Patients' satisfaction is the most important determinant of treatment success. Hence, the main purpose of treating haemorrhoids is to eliminate symptoms, minimising recurrences and produces the least postoperative pain experience and complications. ${ }^{6,44}$ To date, there is no ultimate treatment that suits all patients with symptomatic haemorrhoids. A reasonable approach would be tailored to the degree of severity and patients expectation. ${ }^{8}$

Medical treatment is considered successful in $78-80 \%$ of lower severity grades of haemorroids. ${ }^{45}$ On the other hand, surgical treatment should be offered when medical therapy failed. Surgery is the primary option in the symptomatic managing third and fourth -degree haemorrhoids, or in patients with acute haemorrhoids that failed to improve with other therapies. ${ }^{6,46-48}$

A study reviewing the management of haemorrhoids was conducted in Italy in the period of 2000 to 2016 in which 32,000 patients were included from 18 colorectal units. For Grade III haemorrhoids, substantial changes in surgeon preferences have occurred in the time frame considered. The study showed that the excisional haemorrhoidectomy remains definitively the most common procedure adopted in Italy. However, its frequency of use fell from about $70 \%$ to less than $60 \%$ in the period between 2006-2010, and then returned to $70 \%$ after $2010.4^{49}$

Although excisional haemorrhoidectomy is the 'gold standard' of surgical treatment for symptomatic haemorrhoids, postoperative pain control following haemorrhoidectomy still appears to be the most 
important motivating factor to drive surgeons for a better choice of techniques. ${ }^{41}$ The important aspect that the surgeons could be focusing now should be optimising the postoperative pain management, by local anaesthetic infiltration, ${ }^{1}$ oral analgesics, and the use of topical muscle relaxants. ${ }^{6}$ Conventionally, NSAIDs and opiates have often been used to control pain, but their use is confined to a short time and is associated with various side effects. A randomised controlled trial concluded that perianal ropivacaine with dexmedetomidine is effective and safe and can be a better alternative strategy for reducing the pain. Dexmedetomidine (Precedex ${ }^{\circledR}$, Hospira, Inc., Lake Forest, IL) is a newly developed selective a2adrenoceptor agonist. ${ }^{50}$ Mesoglycan has also been useful in reducing the incidence of thrombosis and the severity of pain during a digital rectal examination after an open diathermy excisional haemorrhoidectomy at 7-10 days postoperation. ${ }^{51}$ Acupuncture was also proven to be effective and can be safely used to control pain after haemorrhoidectomy. ${ }^{52}$ Many innovative operative modifications have been done to enhance this technique by reducing postoperative pain and accelerating wound recovery. The recommended strategies include the use of energy devices during excisional haemorrhoidectomy, postoperative metronidazole and stool softeners use, intradermal Methylene Blue injection, and topical Glyceryl Trinitrate (GTN) ointment application. ${ }^{37}$

Randomised controlled trial comparing stapled haemorrhoidopexy with excisional haemorrhoidectomy for grade II to IV haemorrhoids showed to be less painful with similar complication rate. Quality of life was significantly better among the conventional group over the 2-year follow up, and it is significantly costlier to perform the stapled haemorrhoidectomy procedure. ${ }^{8}$ It may now be the time to accept that stapled haemorrhoidopexy has done its role in directing our understanding to the fact that modern surgical treatment of haemorrhoids should avoid excision of the anorectal skin, which is the main contributor to the postoperative pain associated with standard haemorrhoidectomy. The haemorrhoid treatment intervention should now be focused above the dentate line. We should now move on to our next quest in finding the ideal treatment for one of the oldest medical conditions known. ${ }^{7}$ Transanal haemorrhoidal dearterilization and stapled haemorrhoidopexy produces lesser postoperative pain and faster recovery, but they are associated with higher recurrence rates. ${ }^{53}$

Stapled haemorrhoidopexy is the least painful procedure among the operative modalities. Both stapled haemorrhoidopexy and excisional haemorrhoidectomy are equally safe. However, the excisional haemorrhoidectomy is known to produce less recurrence but more post-operative pain in contrast to the much lesser pain by the stapled haemorrhoidopexy. ${ }^{43}$ The reported recurrence rate of stapler haemorrhoidopexy is $5.7-20.7 \%{ }^{40,42,43}$ It seems obvious that stapled haemorrhoidopexy might be costly. This cost-effectiveness issue should be elaborated, considering the cost of pain-control management and loss of days at work produced by its excisional counterparts. In this aspect, a more extensive direct comparison cost-effectiveness study is needed to guide surgeons' choice. ${ }^{46,54,55}$ Perhaps stapled haemorrhoidopexy should not be recommended for patients who have symptomatic haemorrhoids with excessive external components.

With regards to Haemorrhoidal Artery Ligation (HAL), it has so far proven to be a painless, safe, and efficacious method to treat haemorrhoids, particularly if bleeding is the main complaint. Combining HAL with a recto-anal repair (HAL-RAR) resolves the prolapsed component. To most effective surgical treatment should be tailored to the individual patients' clinical symptoms. ${ }^{56,57}$ When compared with stapled haemorrhoidopexy, HAL resulted in relatively less discomfort; however, the procedure took longer, more expensive and resulted in a higher long term recurrence rate. ${ }^{8}$

With regards to office procedures, haemorrhoidal band ligation is a safe and effective procedure for patients presented with bleeding as the main symptom ${ }^{58}$. It is an option for patients with coagulation disorder, or they are not willing for surgical procedure. No major complications usually encountered, and most patients remain asymptomatic after one session of banding. ${ }^{59}$ When compared with HAL, it is associated with less pain, less cost, and not technically demanding. ${ }^{60}$ While the rubber band ligation may no longer be popular nowadays, it is perhaps convenient to be used in bleeding grade I and II haemorrhoids. 


\section{CONCLUSION}

Tailoring of treatment to the grade of haemorrhoids and patients' symptoms should be a priority. Diet control and lifestyle should be introduced to all patients. Flavonoids or other medical treatment may be helpful in some patients. An office procedure may be tried for those where symptoms persist, and those with grade II haemorrhoids with a course of rubber band ligation. Failing which, surgical intervention with the least invasive procedure is the next choice. The most effective therapy for grade III and IV haemorrhoids appears to be excisional haemorrhoidectomy with the use of one of the energy devices. In open haemorrhoidectomy, there are reasonable evidence for the use of metronidazole postoperatively along with other effective and extensive pain control management. Treatments targeting at ligating of haemorrhoidal pedicles such as doppler-guided haemorrhoidal artery ligation and stapled haemorrhoidopexy are reserved for cases with circumferential prolapse where excisional haemorrhoidectomy to preserve adequate mucocutaneous bridges is difficult. Laser haemorrhoidectomy might not be appropriate for these circumferentially prolapsing haemorrhoids.

\section{CONFLICT OF INTEREST}

None Declared.

\section{REFERENCES}

1. Brown, S. R. Haemorrhoids: an update on management. Ther. Adv. Chronic Dis. 101, 2730 (2017).

2. Agbo, S. P. Surgical Management of Hemorrhoids. J. Surg. Tech. Case Rep. 3, 68 (2011).

3. Chen, J. \& You, J. current status of surgical treatment for hemorrhoids-systematic review and metaanalysis. 488-500 (2010).

4. Naderan, M. et al. A Randomized Controlled Trial Comparing Laser Intra-Hemorrhoidal Coagulation and Milligan-Morgan Hemorrhoidectomy. J. Investig. Surg. 30, 325331 (2017).

5. Demir, H., Karaman, K., Ercan, M., Kocer, H. B. \& Celebi, F. Comparison of two procedures for symptomatic hemorrhoidal disease: Ligation under Vision and Ferguson Hemorrhoidectomy - A retrospective cohort study. Pakistan J. Med. Sci. 33, 90-95 (2017).

6. Cerato, M. M., Cerato, N. L., Passos, P., Treigue, A. \& Damin, D. C. Surgical treatment of hemorrhoids: a critical appraisal of the current options. ABCD. Arq. Bras. Cir. Dig. (São Paulo) 27, 66-70 (2014).

7. Aly, E. H. Stapled haemorrhoidopexy: Is it time to move on? Ann. R. Coll. Surg. Engl. 97, 490-493 (2015).

8. Picchio, M., Palimento, D., Attanasio, U. \& Renda, A. Stapled vs open hemorrhoidectomy: long-term outcome of a randomized controlled trial. Int. J. Colorectal Dis. 21, 668-669 (2006).

9. Vidal, B. Y. V. Hemorrhoid Embolization: Does It Last? 17, 82-84 (2018).

10. Sakr, M. Recent advances in the management of hemorrhoids. World J. Surg. Proced. 4, 55 (2014).

11. Sun, Z. \& Migaly, J. Review of Hemorrhoid Disease: Presentation and Management. Clin. Colon Rectal Surg. 29, 22-29 (2016).

12. Lohsiriwat, V. Hemorrhoids: From basic pathophysiology to clinical management. World $\mathrm{J}$ Gastroenterol 18, 2009-2017 (2012).

13. Margetis, N. Pathophysiology of internal hemorrhoids. Ann Gastroenterol 32, 1-9 (2019).

14. Lohsiriwat, V. Treatment of hemorrhoids: A coloproctologist's view. World J Gastroenterol 21, 9245-9252 (2015).

15. Ganz, R. A. The Evaluation and Treatment of Hemorrhoids: A Guide for the Gastroenterologist. Clin. Gastroenterol. Hepatol. 11, 593-603 (2013).

16. Davis, B. R., Lee-Kong, S. A., Migaly, J., Feingold, D. L. \& Steele, S. R. The American Society of colon and rectal surgeons clinical practice guidelines for the management of hemorrhoids. Dis. Colon Rectum 61, 284-292 (2018).

17. Yamana, T. Japanese Practice Guidelines for Anal Disorders I. Hemorrhoids. J. Anus, Rectum Colon 1, 89-99 (2017).

18. Shelygin, Y. et al. Clinical acceptability study of micronized purified flavonoid fraction $1000 \mathrm{mg}$ tablets versus $500 \mathrm{mg}$ tablets in patients suffering acute hemorrhoidal disease. Curr. Med. Res. Opin. 32, 1821-1826 (2016).

19. Gami, B. Hemorrhoids - A Common Ailment Among Adults, Causes \& Treatment: A Review. Int. J. Pharm. Pharm. Sci. 3, 5-13 (2011). 
20. Zagriadskiı̌, E. A., Bogomazov, A. M. \& Golovko, E. B. Conservative Treatment of Hemorrhoids: Results of an Observational Multicenter Study. Adv. Ther. 35, 1979-1992 (2018).

21. Shrivastava, L., Silva Borges, G. da \& Shrivastava, R. Clinical Efficacy of a Dual Action, Topical Anti-edematous and Antiinflammatory Device for the Treatment of External Hemorrhoids. Clin. Exp. Pharmacol. 08, 1-7 (2018).

22. Albuquerque, A. Rubber band ligation of hemorrhoids: A guide for complications. World J Gastrointest Surg 8, 614-620 (2016).

23. Nelson, R. S. et al. Risk of late bleeding following hemorrhoidal banding in patients on antithrombotic prophylaxis. Am. J. Surg. 196, 994-999 (2008).

24. Acheson, A. G. \& Scholefield, J. H. Management of haemorrhoids. 380-383 (2008). doi:10.1136/bmj.39465.674745.80

25. Fernandes, V. \& Fonseca, J. Polidocanol Foam Injected at High Doses with Intravenous Needle: The (Almost) Perfect Treatment of Symptomatic Internal Hemorrhoids. Orig. Artic. GE Port J Gastroenterol 26, 169-175 (2019).

26. Tomiki, Y. et al. Clinical Study Treatment of Internal Hemorrhoids by Endoscopic Sclerotherapy with Aluminum Potassium Sulfate and Tannic Acid. Diagn. Ther. Endosc. 2015, (2015).

27. Rohit, D. K., Thakur, O., Verma, R. S. \& Pandey, G. Effectivity of injection sclerotherapy with sodium tetra decyl sulphate in the management of first and second-degree hemorrhoids. Int. Surg. J. Rohit DK al. Int Surg J 5, 897-903 (2018).

28. Nikshoar, M. R., Maleki, Z. \& Honar, B. N. The Clinical Efficacy of Infrared Photocoagulation Versus Closed Hemorrhoidectomy in Treatment of. Laser Appl. Med. Sci. Res. Cent. 9, 23-26 (2018).

29. Pravin J. Gupta. Novel Approach to Advanced Hemorrhoidal Disease. Rom. J. Gastroenterol. 76, 49-55 (2014).

30. Trompetto, M. et al. Evaluation and management of hemorrhoids: Italian society of colorectal surgery (SICCR) consensus statement. Tech. Coloproctol. 19, 567-575 (2015).

31. Hoyuela, C. et al. HAL-RAR ( Doppler guided haemorrhoid artery ligation with recto-anal repair ) is a safe and effective procedure for haemorrhoids. Results of a prospective study after two-years follow-up. Int. J. Surg. 28, 3944 (2016).

32. Maloku, H., Gashi, Z., Lazovic, R., Islami, H. \& Juniku-Shkololli, A. Laser hemorrhoidoplasty procedure vs open surgical hemorrhoidectomy: A trial comparing 2 treatments for hemorrhoids of third and fourth degree. Acta Inform. Medica 22, 365-367 (2014).

33. Faes, S., Pratsinis, M., Hasler-Gehrer, S., Keerl, A. \& Nocito, A. Short- and long-term outcomes of laser haemorrhoidoplasty for grade II-III haemorrhoidal disease. Colorectal Dis. 21, 689-696 (2019).

34. De Nardi, P. et al. Hemorrhoid laser procedure for second- and third-degree hemorrhoids: results from a multicenter prospective study. Tech. Coloproctol. 20, 455-459 (2016).

35. Giamundo, P. et al. The hemorrhoid laser procedure technique vs rubber band ligation: A randomized trial comparing 2 mini-invasive treatments for second- and third-degree hemorrhoids. Dis. Colon Rectum 54, 693-698 (2011).

36. Wang, J. Y. et al. Prospective, randomized, controlled trial of Starion ${ }^{T M}$ vs. Ligasure ${ }^{T M}$ hemorrhoidectomy for prolapsed hemorrhoids. Dis. Colon Rectum 50, 1146-1151 (2007).

37. Heng, G. \& Tan, K. Y. Excision Hemorrhoidectomy: New Methods to Improve the Outcomes of an Old Technique. 7, 8-11 (2016).

38. Chugh, A., Singh, R. \& Agarwal, P. N. Management of Hemorrhoids. Indian J. Clin. Pract. 25, 577-580 (2014).

39. Watson, A. J. M. et al. A pragmatic multicentre randomised controlled trial comparing stapled haemorrhoidopexy with traditional excisional surgery for haemorrhoidal disease: The eTHoS study. Health Technol. Assess. (Rockv). 21, 1223 (2017).

40. Lee, K. C. et al. Meta-analysis of randomized controlled trials comparing outcomes for stapled hemorrhoidopexy versus LigaSure hemorrhoidectomy for symptomatic hemorrhoids in adults. Int. J. Surg. 11, 914-918 (2013).

41. Evans, C. F. M., Hyder, S. A. \& Middleton, S. B. Modern surgical management of haemorrhoids. 139-142

42. Giordano, P., Gravante, G., Sorge, R., Ovens, 
L. \& Nastro, P. Long-term Outcomes of Stapled Hemorrhoidopexy vs Conventional Hemorrhoidectomy. Arch. Surg. 144, 266 (2009).

43. Tjandra, J. J. \& Chan, M. K. Y. Systematic review on the procedure for prolapse and hemorrhoids (stapled hemorrhoidopexy). Dis. Colon Rectum 50, 878-892 (2007).

44. Song, S. \& Kim, S. Coloproctology Optimal Treatment of Symptomatic Hemorrhoids. J Korean Soc Coloproctol 27, 277-281 (2011).

45. Coulibaly, A. et al. The Haemorrhoids ' Pathology: Epidemiological, Diagnostic, Therapeutic and Evolutionary Aspects. Open J. Gastroenterol. 06, 343-352 (2016).

46. Watson, A. J. M. M. et al. Comparison of stapled haemorrhoidopexy with traditional excisional surgery for haemorrhoidal disease ( eTHoS ): a pragmatic, multicentre, randomised controlled trial. Lancet $388,2375-$ 2385 (2006).

47. Kendirci, M., Șahiner, I. T., Şahiner, Y. \& Güney, G. Comparison of Effects of VesselSealing Devices and Conventional Hemorrhoidectomy on Postoperative Pain and Quality of Life. Med. Sci. Monit. 24, 2173-2179 (2018).

48. Woodward, T., Crook, J. E., Raimondo, M. \& Wallace, M. Improving complete EMR of colorectal neoplasia: A randomized trial comparing snares and injectate in the resection of large sessile colon polyps. Gastrointest. Endosc. 81, 673-681 (2015).

49. Altomare, D. F. et al. Surgical management of haemorrhoids: an Italian survey of over 32000 patients over 17 years. Color. Dis. 1117-1124 (2018). doi:10.1111/codi.14339

50. Gupta, P. J. Novel approach to advanced hemorrhoidal disease. Rom. J. Gastroenterol. 14, 361-6 (2005).

51. Gallo, G. et al. Efficacy of Mesoglycan in Pain Control after Excisional Hemorrhoidectomy: A Pilot Comparative Prospective Multicenter Study. Gastroenterol. Res. Pract. 2018, 1-8 (2018).

52. Wu, J. et al. Effect of acupuncture on posthemorrhoidectomy pain : a randomized controlled trial. J. Pain Res. 11, 1489-1496 (2018).

53. Simillis, C. et al. Systematic review and network meta-analysis comparing clinical outcomes and effectiveness of surgical treatments for haemorrhoids. 1603-1618 (2015). doi:10.1002/bjs.9913

54. Chen, J. \& You, J. Current Status of Surgical Treatment for Hemorrhoids - Systematic Review and Meta-analysis. 488-500 (2010).

55. Rivadeneira, D. E. et al. Practice parameters for the management of hemorrhoids (Revised 2010). Dis. Colon Rectum 54, 1059-1064 (2011).

56. Boarini, P. et al. Coloproctology LASER hemorrhoidal dearterialization. J. Coloproctology 7, 38-43 (2017).

57. Jeong, W. J. et al. Coloproctology One Year Follow-up Result of Doppler-guided Hemorrhoidal Artery Ligation and Recto-Anal Repair in 97 Consecutive Patients Coloproctology. J Korean Soc Coloproctol 27, 298-302 (2011).

58. Ramzisham, A. R. M., Sagap, I., Nadeson, S., Ali, I. M. \& Hasni, M. J. Prospective randomized clinical trial on suction elastic band ligator versus forceps ligator in the treatment of haemorrhoids. Asian J. Surg. 28, 241-245 (2005).

59. Kim, B. G. \& Kang, H. The effect of preemptive perianal ropivacaine and ropivacaine with dexmedetomidine on pain after hemorrhoidectomy: a prospective, randomized, double-blind, placebo-controlled study. Indian J. Surg. 76, 49-55 (2014).

60. Steven R Brown, James $P$ Tiernan, Angus $J M$ Watson, Katie Biggs, Neil Shephard, Allan J Wailoo, Mike Bradburn, Abualbishr Alshreef, D. $\mathrm{H}$. et al. Haemorrhoidal artery ligation versus rubber band ligation for the management of symptomatic second-degree and third-degree haemorrhoids (HubBLe): a multicentre, openlabel, randomised controlled trial. Lancet 388, 356-364 (2016). 